Pacific Journal of Mathematics

CONCERNING HEREDITARILY INDECOMPOSABLE

R. B No 


\title{
CONCERNING HEREDITARILY INDECOMPOSABLE CONTINUA
}

\author{
R. H. BING
}

1. Introduction. A continuum is indecomposable if it is not the sum of two proper subcontinua. It is hereditarily indecomposable if each of its subcontinua is indecomposable. In [3] Knaster gave an example of a hereditarily indecomposable continuum which was not a point. In this paper we study some properties of the Knaster example and describe some other hereditarily indecomposable continua.

2. Chained hereditarily indecomposable continua are homeomorphic. The hereditarily indecomposable continuum given [3] by Knaster was a plane continuum which was described in terms of covering bands. For each positive number $\epsilon$, it could be covered by an $\epsilon$-chain. Moise used [5] a hereditarily indecomposable continuum to exhibit a continuum which was topologically equivalent to each of its nondegenerate subcontinua. He called it a pseudo-arc and noted that it was similar (if not in fact topologically equivalent) to Knaster's example. It could be chained. Bing used [2] such a continuum as an example of a homogeneous plane continuum. Anderson showed [1] that the plane is the sum of a continuous collection of such continua. Theorem 1 reveals that all of these continua are topologically equivalent.

We follow the definitions used in [2]. In particular, we recall the following. A chain $D=\left[d_{1}, d_{2}, \cdots, d_{n}\right]$ is a collection of open sets $d_{1}, d_{2}, \cdots, d_{n}$ such that $d_{i}$ intersects $d_{j}$ if and only if $i$ is equal to $j-1, j$, or $j+1$. If the links are of diameter less than $\epsilon$, the chain is called an $\epsilon$-chain. We do not suppose that the links of a chain are necessarily connected.

If the chain $E=\left[e_{1}, e_{2}, \cdots, e_{n}\right]$ is a refinement of the chain $D=\left[d_{1}, d_{2}\right.$, $\left.\cdots, d_{m}\right], E$ is called crooked in $D$ provided that if $k-h>2$ and $e_{i}$ and $e_{j}$ are links of $E$ in links $d_{h}$ and $d_{k}$ of $D$, respectively, then there are links $e_{r}$ and $e_{s}$ of $E$ in links $d_{k-1}$ and $d_{h+1}$, respectively, such that either $i>r>s>j$ or $i<$ $r<s<j$.

EXAMPLE 1. The pseudo-arc. The following description of a chained hereditarily indecomposable continuum appeared in [2] and is much like one given

Received June 1, 1950.

Pacific J. Math. 1 (1951), 43-51. 
earlier in $[5]$. In the plane let $D_{1}, D_{2}, \cdots$ be a sequence of chains between the distinct points $p$ and $q$ such that for each positive integer $i$, (a) $D_{i+1}$ is crooked in $D_{i}$, (b) no link of $D_{i}$ has a diameter of more than $1 / i$, and (c) the closure of each link of $D_{i+1}$ is a compact subset of a link of $D_{i}$. The common part of the sum of the links of $D_{1}$, the sum of the links of $D_{2}, \cdots$ is a pseudo-arc. That it is hereditarily indecomposable is shown in [2] and [5].

A continuum can be chained if for each positive number $\epsilon$, the continuum can be covered by an $\epsilon$-chain. A composant of a continuum $W$ is a set $H$ such that for some point $p$ of $\mathbb{W}, H$ is the sum of all proper subcontinua of $W$ containing $p$. We recall that a nondegenerate indecomposable compact continuum has uncountably many composants and no two of its composants intersect each other. The following result holds in a metric space.

THEOREM 1. The compact nondegenerate hereditarily indecomposable continua $M$ and $M^{\prime}$ are homeomorphic if each can be chained.

In fact, if $p$ and $q$ are points of different composants of $M$ while $p^{\prime}$ and $q^{\prime}$ are points of different composants of $M^{\prime}$, there is a homeomorphism carrying $M$ into $M^{\prime}, p$ into $p^{\prime}$, and $q$ into $q^{\prime}$.

Proof. Since $M$ can be chained, there is a sequence $C_{1}, C_{2}, \cdots$ such that $C_{i}$ is a $1 / i$-chain covering $M$, each element of $C_{i}$ intersects $M$, and $C_{i+1}$ is a refinement of $C_{i}$.

First, we show that there is an integer $n_{2}$ so large that $C_{n_{2}}$ is crooked in $C_{1}=\left[c_{1,1}, c_{1,2}, \cdots, c_{1, t_{1}}\right]$. If this were not true there would be elements $c_{1, h}$ and $c_{1, k}$ of $C_{1}$ such that $k-h>2$ and for infinitely many integers $m$, $C_{m}=\left[c_{m, 1}, c_{m, 2}, \cdots, c_{m, t_{m}}\right]$ would have two links $c_{m, i}$ and $c_{m, j}$ in $c_{1, h}$ and $c_{1, k}$ respectively such that if $c_{m, r}$ is in $c_{1, k-1}$ and between $c_{m, i}$ and $c_{m, j}$, then there is not a link of $C_{m}$ in $c_{1, h+1}$ which is between $c_{m, r}$ and $c_{m, j}$. Denote by $W_{m}$ the sum of $c_{m, i}, c_{m, r}$, and the elements of $C_{m}$ between them, where we suppose that no element of $C_{m}$ in $c_{1, k-1}$ is between $c_{m, i}$ and $c_{m, r}$. Let $V_{m}$ be the sum of $c_{m, r}, c_{m, j}$, and the elements of $C_{m}$ between them.

Let $a_{1}, a_{2}, \cdots$ be an increasing sequence of integers such that both $\mathbb{W}_{a_{1}}$, $W_{a_{2}}, \cdots$ and $V_{a_{1}}, V_{a_{2}}, \cdots$ converge. But the limiting set $W$ of $W_{a_{1}}, W_{a_{2}}, \cdots$ is a continuum which intersects $\bar{c}_{1, h}$ but not $\bar{c}_{1, k}$. Also, the limiting set $V$ of $V_{a_{1}}, V_{a_{2}}, \cdots$ is a continuum which intersects $\bar{c}_{1, k}$ but not $\bar{c}_{1, h}$. Hence the assumption that there is no integer $n_{2}$ such that $C_{n_{2}}$ is crooked in $C_{1}$ has led to the contradiction that the hereditarily indecomposable continuum $M$ contains the 
decomposable continuum $W+V$.

Hence, there is a subsequence $C_{n_{1}}, C_{n_{2}}, C_{n_{3}}, \cdots$ of $C_{1}, C_{2}, C_{3}, \cdots$ such that $C_{n_{\imath+1}}$ is crooked in $C_{n_{i}}$.

Let $p$ and $q$ be points belonging to different composants of $M$. Then for each integer $j$, there is an integer $k$ greater than $j$ such that the subchain of $C_{n_{k}}$ from $p$ to $q$ has a link that intersects the first link of $C_{n_{j}}$ and has a link that intersects the last link of $C_{n}$. To see that this is so, let $W_{i}$ be the sum of the links of the subchain of $C_{n_{i}}$ from $p$ to $q$. Since the limiting set of each subsequence $W_{1}, W_{2}$, -. is a continuum in $M$ containing $p+q$, each such limiting set is $M$. Hence, some $\mathbb{W}_{k}(k>j)$ intersects both the first and last links of $C_{n_{k}}$, and the subchain of $C_{n_{k}}$ corresponding to $W_{k}$ has links intersecting the first and last links of $C_{n_{j}}$

We find from Theorem 4 of [2] that there is a chain $E_{j}$ such that the first link of $E_{j}$ contains $p$, the last contains $q, E_{j}$ is a consolidation of $\mathrm{C} n_{j}$, while each link of $E_{j}$ lies in the sum of two adjacent links of $C_{n_{j}}$ and is therefore of diameter less than $2 / j$. Hence, there is no loss of generality in supposing that each of the chains $C_{1}, C_{2}, \cdots$ is from $p$ to $q$. Therefore, there is a sequence $D_{1}, D_{2}, \cdots$ of chains from $p$ to $q$ such that for each positive integer $i$, (a) $D_{i+1}$ is crooked in $D_{i}$, (b) the closure of each link of $D_{i+1}$ is a subset of a link of $D_{i}$, (c) no link of $D_{i}$ has a diameter of more than $1 / i$, and (d) $M$ is the common part of the sum of the links of $D_{1}$, the sum of the links of $D_{2}, \cdots$.

Similarly, we find that if $p^{\prime}$ and $q^{\prime}$ are points of different composants of $M^{\prime}$, there is a sequence $D_{1}^{\prime}, D_{2}^{\prime}, \cdots$ of chains from $p^{\prime}$ to $q^{\prime}$ such that (a) $D_{i+1}^{\prime}$ is crooked in $D_{i}^{\prime}$, (b) the closure of each element of $D_{i+1}^{\prime}$ is a subset of an element of $D_{i}^{\prime}$, (c) no element of $D_{i}^{\prime}$ has a diameter of more than $1 / i$, and (d) $D_{i}$ covers $M^{\prime}$. Theorem 12 of [2] shows that there is a homeomorphism that carries $M$ into $M^{\prime}$, $p$ into $p^{\prime}$, and $q$ into $q^{\prime}$.

The preceding theorem shows that $M$ is homogeneous and homeomorphic with each of its nondegenerate subcontinua. It also reveals that the continua studied by Knaster [3], Moise [5], Bing [2], and Anderson [1] are all topologically equivalent and are pseudo-arcs.

QUESTION. It would be interesting to know if each nondegenerate bounded hereditarily indecomposable plane continuum which does not separate the plane is homeomorphic to $M$. This question would be answered in the affirmative if it were shown that each bounded atriodic plane continuum which does not separate the plane can be chained (see Section 6, below).

3. Most continua are pseudo-arcs. Mazurkiewicz showed [4] that the continua 
contained in a circle plus its interior which were not hereditarily indecomposable were of the first category. We go even further to show that those which are not pseudo-arcs are of the first category.

The space of compact continua in the metric space $S$ is the metric space $C(S)$ whose points are the compact continua of $S$, where the distance between two elements $g_{1}, \mathrm{~g}_{2}$ of $C(S)$ is the Hausdorff distance between them in $S$, namely L. U.B. (distance from $x$ to $g_{i}$ in $S$ ), $x \in g_{1}+g_{2} ; i=1,2$. By saying that most compact continua of $S$ have a certain property, we mean that there is a dense inner limiting $\left.{ }^{(} G \delta\right)$ subset $\mathbb{W}$ of $C(S)$ such that each element of $W$ has the property (when regarded as a continuum in $S$ ). The collection of continua of $S$ with this property is said to be of the second category.

The following theorem holds in a space $S$ which is either a Hilbert space or a Euclidean $n$-space $(n>1)$.

\section{THEOREM 2. Most compact continua are pseudo-arcs.}

Proof. Let $F_{i}$ be the collection of all compact continua $f$ in $S$ such that $f$ can not be covered by a $1 / i$-chain. If $f_{1}, f_{2}, \cdots$ is a sequence of elements of $F_{i}$ converging to a compact continuum $f_{0}, f_{0}$ is an element of $F_{i}$ because if a $1 / i$-chain covers $f_{0}$, it covers some $f_{j}$. Hence, $F_{i}$ is closed in $C(S)$.

Let $G_{i}$ be the set of all compact continua $K$ such that $K$ contains a subcontinuum $K^{\prime}$ which is the sum of two continua $K_{1}$ and $K_{2}$ such that $K_{1}$ contains a point at a distance (in $S$ ) of $1 / i$ or more from $K_{2}$, while $K_{2}$ contains a point at a distance of $1 / i$ or more from $K_{1}$. Then $G_{i}$ is a closed subset of $C(S)$. Furthermore, the collection of points of $S$ is closed in $C(S)$.

The collection of all pseudo-arcs is dense in $C(S)$. For suppose that $g$ is an element of $C(S)$ and $\epsilon$ is a positive number. There is a broken line $a b$ whose distance (in $C(S)$ ) from $g$ is less than $\epsilon / 2$. Let $D$ be an $\epsilon / 2$-chain from $a$ to $b$ covering $a b$ such that each element of $D$ is the interior of a sphere. There is a pseudo-arc $h$ containing $a+b$ which is covered by $D$. The distance from $g$ to $h$ is less than $\epsilon$ in $C(S)$.

Each element of $C(S)$ not belonging to $\Sigma F_{i}$ can be chained and each element of $C(S)$ not belonging to $\Sigma G_{i}$ is hereditarily indecomposable. Let $W$ be the set of all elements $g$ of $C(S)$ such that $g$ is not a point of $S, g$ is not an element of any $F_{i}$, and $g$ is not an element of any $G_{i}$. By Theorem 1, each element of $W$ is topologically equivalent to $M$. However, $W$ is a dense inner limiting subset of $C(S)$.

4. Decomposition of a pseudo-arc. Like a simple closed curve, the pseudo-arc 
is homogeneous; and like an arc it is homeomorphic with each of its nondegenerate subcontinua. We now show that like both a simple closed curve and an arc, it is topologically equivalent to itself under a nontrivial monotone decomposition.

THEOREM 3. If an upper semi-continuous collection of continua fills a chained compact continuum, the resulting decomposition space is chained.

Proof. Suppose $G$ is an upper semi-continuous collection of continua filling the chained continuum $M$ and that the resulting decomposition space $M^{\prime}$ is given a metric. We show that for each positive number $\epsilon$, an $\epsilon$-chain in $M^{\prime} \operatorname{covers} M^{\prime}$.

Let $\delta$ be a positive number so small that if two elements of $G$ are no farther apart in $M$ than $\delta$, then the points in $M^{\prime}$ corresponding to them are no farther apart than $\epsilon / 5$ in $M^{\prime}$. Let $[c(1), c(2), \cdots, c(n)]$ be a $\delta$-chain covering $M$. Let $n_{1}=1$, $n_{2}, n_{3}, \cdots, n_{j}=n$ be a monotone increasing sequence of integers such that an element of $G$ intersects $c\left(n_{i}\right)$ and $c\left(n_{i+1}\right)$, but none intersects $c\left(n_{i}\right)$ and $c\left(n_{i+1}+1\right)$. Denote by $D(i, j)$ the open subset of $M^{\prime}$ consisting of those points corresponding to elements of $G$ which are covered by $c(i)+c(i+1)+\cdots+c(j)$. Then

$$
\begin{aligned}
& D\left(n_{1}, n_{5}\right), D\left(n_{4}, n_{8}\right), D\left(n_{7}, n_{11}\right), \cdots, D\left(n_{3 k+1}, n_{j}\right) \text {, } \\
& j-5 \leq 3 k+1<j-3
\end{aligned}
$$

is an $\epsilon$-chain covering $M^{\prime}$.

The following result follows from Theorems 1 and 3 .

THEOREM 4. If $M$ is a pseudo-arc and $G$ is an upper semi-continuous collection of proper subcontinua of $M$ filling $M$, the resulting decomposition space is topologically equivalent to $M$.

5. Other types of hereditarily indecomposable plane continua. The pseudo-arc can be imbedded in the plane. We now show that there are nondegenerate hereditarily indecomposable plane continua which are not topologically equivalent to the pseudo-arc. In fact, there are as many topologically different hereditarily indecomposable plane continua as there are plane continua.

A method which differs from the one below of showing that there are topologically different nondegenerate plane continua is to consider in 3-space the intersection of a plane with a hereditarily indecomposable continuum which separates 3-space. That there are such continua in 3-space will be shown in my paper, Higher dimensional hereditarily indecomposable continua, which proves that there are hereditarily indecomposable continua of all dimensions. 
EXAMPLE 2. A hereditarily indecomposable continuum that separates the plane. A circular chain differs from a chain in that its first and last links intersects each other. In the plane let $D_{1}, D_{2}, \cdots$ be a sequence of circular chains such that (a) each element of $D_{i}$ is the interior of a circle of diameter less than $\mathrm{l} / i$, (b) the closure of each element of $D_{i+1}$ lies in an element of $D_{i}$, (c) the sum of the elements of $D_{i}$ is topologically equivalent to the interior of an annulus ring, (d) each complementary domain of the sum of the elements of $D_{i+1}$ contains a complementary domain of the sum of the elements of $D_{i}$, and (e) if $E_{i}$ is a proper subchain of $D_{i}$ and $E_{i+1}$ is a subchain of $D_{i+1}$ contained in $E_{i}$, then $E_{i+1}$ is crooked in $E_{i}$. Condition (c) is superfluous since it follows from condition (a). The required continuum $M$ is the intersection of the sum of the elements of $D_{1}$, the sum of the elements of $D_{2}, \cdots$.

Suppose that a chain $D_{i}=\left[d_{1}, d_{2}, \cdots, d_{n}\right]$ satisfying the preceding conditions has been found. To see that there is a chain $D_{i+1}$ satisfying the required conditions, it might be convenient first to consider a chain $D_{i}^{\prime}=\left[d_{1}^{\prime}, d_{2}^{\prime}, \cdots\right.$, $\left.d_{3 n}^{\prime}\right]$ in $D_{i}$ which follows the following pattern: $d_{1}^{\prime}, d_{n+1}^{\prime}, d_{2 n+1}^{\prime}$ are subsets of $d_{1} ; d_{2}^{\prime}, d_{n+2}^{\prime}, d_{3 n}^{\prime}$ are subsets of $d_{2} ; d_{3}^{\prime}, d_{n+3}^{\prime}, d_{3 n-1}^{\prime}$ are subsets of $d_{3} ; \cdots$; $d_{n}^{\prime}, d_{2 n}^{\prime}, d_{2 n+1}^{\prime}$ are subsets of $d_{n}$. Roughly speaking, $D_{1}^{\prime}$ goes through $D_{i}$ twice in one direction and once in the opposite direction. Then the circular chain $D_{i+1}$ satisfying the required conditions is the sum of two chains one of which is crooked in $\left[d_{1}^{\prime}, d_{2}^{\prime}, \cdots, d_{2 n+1}^{\prime}\right]$ and the other of which is crooked in

$$
\left[d_{2 n+1}^{\prime}, d_{2 n+2}^{\prime}, \cdots, d_{3 n}^{\prime}, d_{i}^{\prime}\right] \text {. }
$$

That $M$ separates the plane follows from conditions (c) and (d). We show that it is hereditarily indecomposable by showing that each of its proper subcontinua is indec omposable.

Suppose $M^{\prime}$ is a proper subcontinuum of $M$. Let $m$ be an integer so large that some element of $D_{m}$ does not intersect $M^{\prime}$. If $E_{k}(k \geq m)$ is the collection of elements of $D_{k}$ which intersect $M^{\prime}$, then $E_{m}, E_{m+1}, \cdots$ is a sequence of chains such that $E_{k+1}$ is crooked in $E_{k}$. If $M^{\prime}$ were the. sum of two proper subcontinua $H$ and $K$, there would be a point $p$ of $H$; a point $q$ of $K$, and an integer $w$ such that the distances from $p$ to $K$ and from $q$ to $H$ are each greater than 2/w. Suppose $e_{i}$ and $e_{j}$ are elements of $E_{w+1}$ containing $p$ and $q$ respectively. Since $E_{w+1}$ is crooked in $E_{w}$, there are elements $e_{r}$ and $e_{s}$ in $E_{w+1}$ such that $e_{r}$ separates $e_{i}$ from $e_{s}$ in $E_{w+1}$, each point of $e_{s}$ is nearer than $2 / w$ to $p$, and each point of $e_{r}$ is nearer to $q$ than $2 / w$. Then $H$ would not be connected because it has a point in $e_{s}$, a point in 
$e_{i}$, but none in $e_{r}$.

Questions. Theorem 1 shows that if $M_{1}$ and $M_{2}$ are two nondegenerate chained hereditarily indecomposable continua, they are topologically equivalent and each is homogeneous. Suppose $W_{1}$ and $W_{2}$ are two continua, each defined as described in Example 2. It would be interesting to know if $W_{1}$ would necessarily be homeomorphic to $W_{2}$. Also, is any such continuum homogeneous?

EXAMPLE 3. Another hereditarily indecomposable continuum. The following example of a hereditarily indecomposable plane continuum is somewhat of a combination of Examples 1 and 2.

If $\mathbb{W}$ is a nondegenerate indecomposable plane continuum, it contains a nondegenerate subcontinuum $H$ such that no point of $H$ is accessible from the complement of $W$. To see that this is true, consider two parallel lines $L_{1}$ and $L_{2}$, each of which separates $W$. Let $G$ be an uncountable collection of mutually exclusive subcontinua of $W$ each irreducible from $L_{1}$ to $L_{2}$, and let $K$ be the sum of $L_{1}, L_{2}$, and the closure of the sum of the elements of $G$. If $D$ is a complementary domain of $K$ between $L_{1}$ and $L_{2}$, its closure does not intersect three elements of $G$. Hence, some element of $G$ is not accessible from any complementary domain of $K$ between $L_{1}$ and $L_{2}$. If $H$ is a subcontinuum of this element of $G$ which does not intersect $L_{1}+L_{2}$, no point of $H$ is accessible from the complement of $\mathbb{W}$.

If $W_{0}^{\prime}$ is a nondegenerate hereditarily indecomposable plane continuum, there is a point $p$ of $W_{0}$ such that if $W^{\prime}$ is a nondegenerate subcontinuum of $W_{0}$ containing $p$, then $p$ is not accessible from the complement of $W^{\prime}$. To find such a point, let $W_{1}, W_{2}, \cdots$ be a sequence of continua such that $W_{i}$ is a subcontinuum of $W_{i-1}$, no point of $W_{i+1}$ is accessible from the complement of $W_{i}$, and $W_{i}$ is of diameter less than $1 / i$. Then $W_{0} \cdot W_{1} \cdot W_{2}^{\prime} \cdot \cdots$ is a point $p$; and if $W^{\prime}$ is a nondegenerate subcontinuum of $W_{0}$ containing $p$, it contains one of the $W_{i}$ 's. Hence, $p$ is not accessible from the complement of $W^{\prime}$.

Let $M_{1}$ be a pseudo-arc in the plane and $M_{2}$ be a hereditarily indecomposable plane continuum as described in Example 2. Let $p$ be a point of $M_{1}$ such that $p$ is not accessible from the complement of any nondegenerate subcontinuum of $M_{1}$. By a theorem of R. L. Moore, there is a continuous transformation $T$ of the plane into itself such that $T^{-1}(p)=\left(M_{2}\right.$ plus its interior $)$ and the inverse of each other point is a point. We show that $M_{3}=\left(T^{-1}\left(M_{1}\right)\right.$ minus the interior of $\left.M_{2}\right)$ is hereditarily indecomposable. That $M_{2}$ and $M_{3}$ are not homeomorphic follows from the fact that $M_{2}$ is irreducible with respect to separating the plane but $M_{3}$ is not. 
If $W$ is a subcontinuum of $M_{3}$ intersecting $M_{2}$ and containing a point of $M_{3}-M_{2}$, it contains $M_{2}$. For suppose that it does not contain some point of $M_{2}$. Then there is an arc $\alpha$ from the exterior of $M_{2}$ to $M_{2}$ that does not intersect $W$. But $T(\alpha)$ would be an arc, revealing that $p$ is accessible from the complement of $T(\mathbb{W})$. This is contrary to the definition of $p$.

Suppose that some subcontinuum $M^{\prime}$ of $M_{3}$ is the sum of two proper subcontinua $H$ and $K$. Since $M_{2}$ is hereditarily indecomposable, $M^{\prime}$ is not a subset of $M_{2}$. Since $M_{1}$ is hereditarily indecomposable, we may suppose that $T(H)=T\left(M^{\prime}\right)$. But $H$ would equal $M^{\prime}$ because $T$ is one-one on the exterior of $M_{2}$ and $H$ contains $M_{2}$ if it intersects it.

A variation of the method used in obtaining Example 3 may be used to get other topologically different hereditarily indecomposable plane continua. Instead of replacing a point of $M_{1}$ by a continuum homeomorphic to $M_{2}$, we can replace each of several points of $M_{1}$ by such a continuum.

THEOREM 5. There are as many topologically different hereditarily indecomposable bounded plane continua as there are real numbers.

Proof. Suppose $n_{1}, n_{2}, \cdots$ is a monotone increasing sequence of positive integers. The collection of such sequences has the power of the continuum. For each such sequence we describe a hereditarily indecomposable plane continuum such that no two of these continua are topologically equivalent.

The hereditarily indecomposable continuum associated with $n_{1}, n_{2}, \cdots$ will have one composant containing exactly $n_{1}$ continua each topologically equivalent to $M_{2}$ of Example 2, another composant containing exactly $n_{2}$ continua each topologically equivalent to $M_{2}, \cdots$; no other composant contains a continuum topologically equivalent to $M_{2}$.

Let $M_{1}$ be a pseudo-arc in the plane. Suppose

$$
p_{1,1}, p_{1,2}, \cdots, p_{1, n_{1}}, p_{2,1}, \cdots
$$

is a converging sequence of different points of $M_{1}$ such that $P_{i, j}$ is not accessible from the complement of any nondegenerate subcontinuum of $M_{1}$ containing it, and $\mathrm{p}_{i, j}$ belongs to the composant containing $p_{r, s}$ if and only if $i=r$.

Suppose $M_{1,1}, M_{1,2}, \cdots, M_{1, n_{1}}, M_{2,1}, \cdots$ is a sequence of mutually exclusive continua in the plane all topologically equivalent to $M_{2}$ of Example 2 such that the sequence converges to a point and each of the continua lies in the exterior 
of each of the others. There is a continuous transformation $T$ of the plane into itself such that $T^{-1}\left(p_{i, j}\right)$ is $\left(M_{i, j}\right.$ plus its interior), and $T^{-1}(q)$ is a point if $q$ is not a $p_{i, j}$. The argument used in Example 3 shows that $M_{3}=\left(T^{-1}\left(M_{1}\right)\right.$ minus the sum of the interiors of the $M_{i, j}$ 's) is a hereditarily indecomposable continuum. Furthermore, one composant of $M_{3}$ contains exactly $n_{1}$ mutually exclusive subcontinua, each topologically equivalent to $M_{2}$, another composant contains exactly $n_{2}$ such subcontinua, $\cdots$, while the other composants of $M_{3}$ contain no such continua.

6. Added in proof. R. D. Anderson answered the question at the end of Section 2 in the negative by announcing at the February, 1951, meeting of the American Mathematical Society in New York that there are nondegenerate bounded hereditarily indecomposable plane continua other than pseudo-arcs which do not separate the plane.

\section{REFERENCES}

1. R. D. Anderson, On collections of pseudo-arcs, Abstract 337t, Bull. Amer. Math. Soc. 56 (1950), 350 .

2. R. H. Bing, A homogeneous indecomposable plane continuum, Duke Math. J. 15 (1948), 729-742.

3. B. Knaster, Un continu dont tout sous-continu est indécomposable, Fund. Math. 3 (1922), 247-286.

4. S. Mazurkiewicz, Sur les continus absolument indécomposable, F und. Math. 16 (1930), 151-159.

5. E. E. Moise, An indecomposable plane continuum which is homeomorphic to each of its nondegenerate subcontinua, Trans. Amer. Math. Soc. 63 (1948), 581-594.

\section{UNIVERSITY OF VIRGINIA AND UNIVERSITY OF WISCONSIN}





\title{
EDITORS
}

\author{
HERBERT BUSEMANN \\ University of Southern California \\ Los Angeles 7, California
}

R. M. RoBINSON

University of California

Berkeley 4, California

E. F. BECKENBACH, Managing Editor

University of California

Los Angeles 24, California

ASSOCIATE EDITORS
R. P. DILWORTH
P. R. HALMOS
BQRGE JESSEN
J. J. STOKER
HERBERT FEDERER
HEINZ HOPF
PAUL LÉVY
E. G. STRAUS
MARSHALL HALL
R. D. JAMES
GEORGE PÓLYA
KÔSAKU YOSIDA

\section{SPONSORS}

UNIVERSITY OF BRITISH COLUMBIA

CALIFORNIA INSTITUTE OF TECHNOLOGY UNIVERSITY OF CALIFORNIA, BERKELEY

UNIVERSITY OF CALIFORNIA, DAVIS

UNIVERSITY OF CALIFORNIA, LOS ANGELES

UNIVERSITY OF CALIFORNIA, SANTA BARBARA

OREGON STATE COLLEGE

UNIVERSITY OF OREGON
UNIVERSITY OF SOUTHERN CALIFORNIA STANFORD UNIVERSITY

WASHINGTON STATE COLLEGE

UNIVERSITY OF WASHINGTON

AMERICAN MATHEMATICAL SOCIETY NATIONAL BUREAU OF STANDARDS, INSTITUTE FOR NUMERICAL ANALYSIS

\section{Vari-Type Composition by \\ Cecile Leonard \\ Ruth Stafford}

With the cooperation of

E. F. Beckenbach

E. G. Straus

Printed in the United States of America by

Edwards Brothers, Inc., Ann Arbor, Michigan

UNIVERSITY OF CALIFORNIA PRESS - BERKELEY AND LOS ANGELES COPYRIGHT 1951 BY PACIFIC JOURNAL OF MATHEMATICS 


\section{Pacific Journal of Mathematics}

\section{Vol. 1, No. $1 \quad$ November, 1951}

Ralph Palmer Agnew, Ratio tests for convergence of series............. 1

Richard Arens and James Dugundji, Topologies for function spaces....... 5

B. Arnold, Distributive lattices with a third operation defined ........... 33

R. Bing, Concerning hereditarily indecomposable continua ........... 43

David Dekker, Generalizations of hypergeodesics ............... 53

A. Dvoretzky, A. Wald and J. Wolfowitz, Relations among certain ranges of vector measures.................................... 59

Paul Erdős, F. Herzog and G. Pirani, Schlicht Taylor series whose convergence on the unit circle is uniform but not absolute .......... 75

Whilhelm Fischer, On Dedekind's function $\eta(\tau) \ldots \ldots \ldots \ldots \ldots \ldots . \ldots 3$

Werner Leutert, The heavy sphere supported by a concentrated force ...... 97

Ivan Niven and H. Zuckerman, On the definition of normal numbers ...... 103

L. Paige, Complete mappings of finite groups .................. 111

Otto Szász, On a Tauberian theorem for Abel summability ............. 117

Olga Taussky, Classes of matrices and quadratic fields ............. 127

F. Tricomi and A. Erdélyi, The asymptotic expansion of a ratio of gamma functions .................................... 133

Hassler Whitney, On totally differentiable and smooth functions ......... 143 